\title{
Seasonal variation in Internet searches for vitamin D
}

\author{
Rebecca J. Moon ${ }^{1,2}$ • Elizabeth M. Curtis ${ }^{1}$ Justin H. Davies ${ }^{2}$ - Cyrus Cooper ${ }^{1,3,4}$ • \\ Nicholas C. Harvey ${ }^{1,3}$
}

Received: 17 January 2017 / Accepted: 28 February 2017 / Published online: 10 March 2017

(C) The Author(s) 2017. This article is published with open access at Springerlink.com

\begin{abstract}
Summary Internet search rates for "vitamin D" were explored using Google Trends. Search rates increased from 2004 until 2010 and thereafter displayed a seasonal pattern peaking in late winter. This knowledge could help guide the timing of public health interventions aimed at managing vitamin D deficiency.

Purpose The Internet is an important source of health information. Analysis of Internet search activity rates can provide information on disease epidemiology, health related behaviors and public interest. We explored Internet search rates for vitamin $\mathrm{D}$ to determine whether this reflects the increasing scientific interest in this topic.

Methods Google Trends is a publically available tool that provides data on Internet searches using Google. Search activity for the term "vitamin D" from 1st January 2004 until 31 st October 2016 was obtained. Comparison was made to other bone and nutrition related terms.

Results Worldwide, searches for "vitamin D" increased from 2004 until 2010 and thereafter a statistically significant $(p<0.001)$ seasonal pattern with a peak in February and nadir
\end{abstract}

Nicholas C. Harvey

nch@mrc.soton.ac.uk

1 MRC Lifecourse Epidemiology Unit, University of Southampton, Southampton, UK

2 Paediatric Endocrinology, University Hospital Southampton NHS Foundation Trust, Southampton, UK

3 NIHR Southampton Nutrition Biomedical Research Centre, University of Southampton and University Hospital Southampton NHS Foundation Trust, Southampton, UK

4 National Institute for Health Research (NIHR) Musculoskeletal Biomedical Research Unit, University of Oxford, Oxford, UK in August was observed. This seasonal pattern was evident for searches originating from both the USA (peak in February) and Australia (peak in August); $p<0.001$ for both. Searches for the terms "osteoporosis", "rickets", "back pain" or "folic acid" did not display the increase observed for vitamin D or evidence of seasonal variation.

Conclusion Public interest in vitamin D, as assessed by Internet search activity, did increase from 2004 to 2010, likely reflecting the growing scientific interest, but now displays a seasonal pattern with peak interest during late winter. This information could be used to guide public health approaches to managing vitamin $\mathrm{D}$ deficiency.

Keywords Vitamin D · Google Trends · Internet · Epidemiology

Background In recent years there has been increasing scientific interest in 25-hydroxyvitamin D [25(OH)D] [1] and its potential role in a wide variety of health outcomes. A large number of observational studies have reported associations between 25(OH)D and malignancy, cardiovascular disease, mental illness, diabetes, obstetric outcomes and all-cause mortality, yet there is a lack of high quality evidence from intervention studies to support a causal role for vitamin $\mathrm{D}$ in outcomes apart from osteomalacia and rickets [2-4]. Nonetheless, both the testing for biochemical 25(OH)D deficiency [5] and prescribing of vitamin D supplementation [6] have increased.

Many national guidelines now provide advice on daily reference intakes (DRIs) for vitamin D and/or suggest vitamin D supplementation for those with risk factors for biochemically low levels of $25(\mathrm{OH}) \mathrm{D}$ [7-9]. Despite this, studies have suggested that the general public have only limited knowledge regarding vitamin $\mathrm{D}[6,10-12]$; in the UK, the National Institute for Health and Care Excellence (NICE) have 
highlighted the importance of increasing public knowledge regarding vitamin D [8]. The Internet is now an important source of health information for the general public, and many individuals will frequently use the Internet as first line when in need of health-related information [13]. As such, analysis of Internet search activity has been shown to provide useful information on the epidemiology of disease [14] and changes in health-related behavior [15], and could potentially guide future public health messages. We therefore undertook this study to explore whether the public interest in vitamin D assessed using Internet search activity reflected the increasing scientific interest in this topic.

\section{Methods}

Data for Internet search activity were obtained using Google Trends (www.google.com/trends). This is a publically searchable database which reports the search activity for a term within Google from January 2004 until the present day. Approximately $65 \%$ of all Internet searches are performed using Google [16]. Absolute numbers of searches per month are not available, but instead the data are presented relative to the month for which search activity was highest on a 0 to 100 scale (100 being the highest month). Search activity can be assessed for worldwide usage, or specific countries of origin. A report detailing the most frequent countries from which the term of interest is searched is also provided. We obtained Google search activity for the search term "vitamin D" for both worldwide and individual countries of origin from 1st January 2004 until 31st October 2016. Where necessary "vitamin D" was translated into the local language for the country of search. The monthly data were downloaded from Google Trends in .csv format to Microsoft Excel on 11th November 2016. Comparison was made to other bone and nutrition-related search terms. SPSS v24 was used to perform cosinor analysis to assess for seasonal variation in search activity. A $p<0.05$ was considered statistically significant.

\section{Results}

Worldwide, Google searches for the term "vitamin D" was highest in February 2016. Figure 1 displays the relative number of searches for "vitamin D" per month compared to the number in February 2016. Internet search activity for "vitamin D" increased from 2004 until 2010. Since 2010, there has been a statistically significant $(p<0.001)$ seasonal pattern to Internet searches for "vitamin D" with a peak in February and nadir in August (Fig. 1). The United States of America (USA) and Australia had the highest number of Internet searches for vitamin $\mathrm{D}$ for the northern and southern hemispheres, respectively. A seasonal pattern in search activity was evident for both the USA and Australia ( $p<0.001$ for both) but this peaked in February in the USA and August in Australia

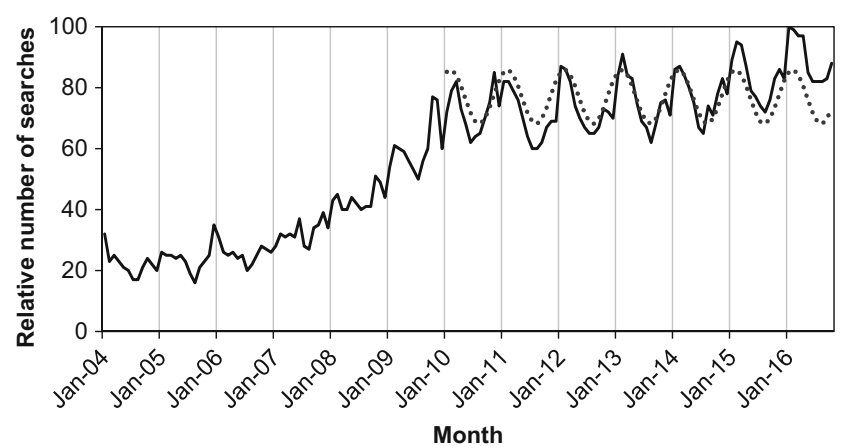

Fig. 1 Google search activity for the term "vitamin D". The dotted line represents the seasonal model generated from these data for January 2010 to October 2016

(Fig. 2). Within Europe, differences in search activity were observed; Sweden and Norway displayed seasonal variation in search activity ( $p<0.05$ for both), whereas searches for "vitamina D" in Spain and Italy occurred less frequently and showed no evidence of seasonality (Fig. 3).

In order to determine if this reflected a seasonal pattern in Internet use, we assessed a number of other musculoskeletal and nutrition-related terms. Google searches for the terms "osteoporosis", "rickets", "back pain" or "folic acid" did not display the increase observed with vitamin D, or evidence of seasonal activity for worldwide searches or from the USA or Australia ( $p<0.05$ for all). Conversely, worldwide searches for "calcium" appeared to have two peaks each year in both October and February. A similar pattern was evident for searches originating from the USA, whereas those from Australia did display seasonal variation peaking in June.

\section{Discussion}

The use of the Internet to access information on vitamin D appeared to increase dramatically between 2004 and 2010 . Since that time, search activity for vitamin D has varied seasonally, peaking in late winter in both the USA and Australia. Although overall search activity using Google has increased

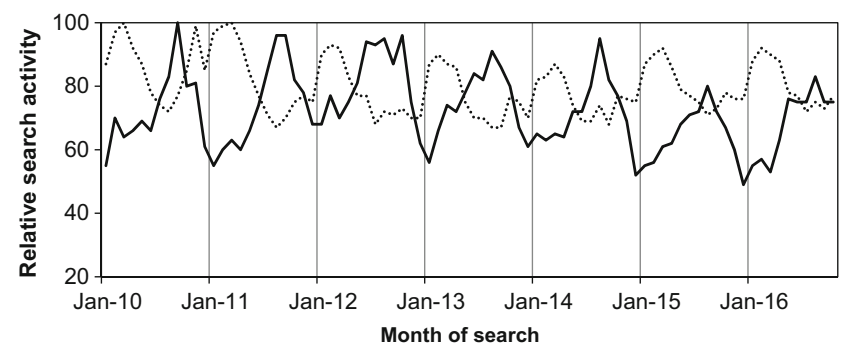

Fig. 2 Google search activity for "vitamin D" for searches originating from the USA (solid line) and Australia (dotted line) 


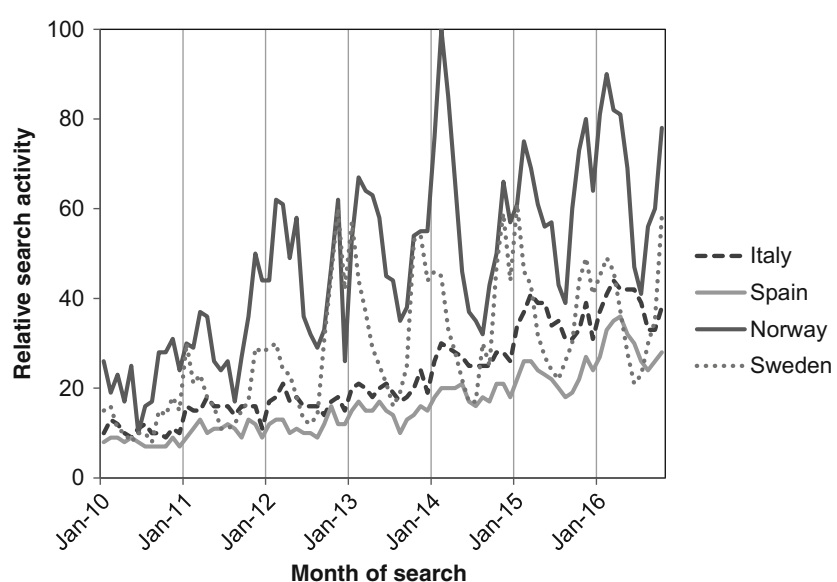

Fig. 3 Google search activity for "vitamin D" in Norway and Sweden compared with searches for "vitamina D" in Spain and Italy. All search counts are relative to the month in which the highest number of searches was made in Norway

exponentially since 2000 [16], a similar trend was not observed for other musculoskeletal and nutrition related terms we explored, suggesting that this rise in general public interest is likely to reflect the increasing scientific interest, publication rate and media coverage.

It is interesting that Internet searches for vitamin D peak in late winter. We believe that this genuinely reflects an increase in public interest in vitamin D during this time rather than simply reflecting increased Internet usage during colder darker winter months; firstly, searches for "osteoporosis", "rickets", "back pain" and "folic acid" did not display seasonal variation and the annual peaks and troughs in searches for "calcium" differed to those for "vitamin D", and secondly, previous studies have, in contrast, demonstrated seasonal variation in searches for restless leg syndrome and foot and ankle pain peaking in the summer months [17, 18]. Furthermore, searches arising from Norway and Sweden did peak in winter, whereas Spain and Italy had far fewer searches for "vitamina D" recorded and no evidence of seasonal variation in this, perhaps suggesting that at more northerly latitudes interest in vitamin D and/or vitamin D deficiency is greater. One possible explanation for this winter peak could be that individuals with some knowledge on the seasonal variation in vitamin D status are seeking further information to attribute vitamin D to symptoms experienced in winter months, as many people will now obtain health information from the internet prior to attending a medical practitioner [13]. Alternatively, information seeking behavior could be secondary to a health professional suggesting $25(\mathrm{OH}) \mathrm{D}$ testing or vitamin D supplementation. These have both increased in recent years $[5,6]$, but to our knowledge, seasonal variation in requests for $25(\mathrm{OH}) \mathrm{D}$ assessment or prescriptions for vitamin $\mathrm{D}$ has not been investigated. Nonetheless, this observation of seasonal variation in Internet search activity may have important implications for public health approaches to improving vitamin D status. In particular, if knowledge seeking behaviors with regard to vitamin D are not occurring until late winter, then the opportunity to prevent biochemically low $25(\mathrm{OH}) \mathrm{D}$ during the winter months is missed. Jha et al. showed that Google search activity for Fosamax peaked following significant coverage of the drug in the popular media [19], highlighting that such an approach could be used to promote interest and knowledge seeking behaviors for a specific topic. As such, timing vitamin D related health messages for early autumn might encourage supplementation usage and prevent the winter nadir in $25(\mathrm{OH}) \mathrm{D}$, thus suggesting the need to work together with the popular media industry to ensure that information is promoted at the most useful time of the year.

The interpretation of these data is not without limitations. Demographic information on those conducting the searches is not available, and individuals who use the Internet for information seeking may not be representative of the general population or those at risk of vitamin D deficiency. In particular, although Internet usage is increasing amongst older individuals, it remains markedly lower than in younger adults [20]. Nonetheless, this is a population that has actively sought to inform themselves and is therefore likely to be receptive to public health education. However, we do not know which websites were accessed from the Google search, and importantly what messages they promote. Furthermore, it is only possible to speculate on the reason for the searches being undertaken. Finally, this approach can only assess internet searches performed using Google and no other search engines; in particular the use of other search engines may vary between countries, but worldwide Google does account for approximately $65 \%$ of all Internet searches [16].

In conclusion, the public interest in vitamin $\mathrm{D}$, as assessed by Internet search activity, initially appeared to mirror the increase in scientific interest, but now displays a seasonal pattern with peak interest during late winter. This information could be used to guide public health approaches to managing vitamin $\mathrm{D}$ deficiency.

Acknowledgements This work was supported by grants from the Medical Research Council, British Heart Foundation, Arthritis Research UK, National Osteoporosis Society, International Osteoporosis Foundation, National Institute for Health Research (NIHR) Southampton Biomedical Research Centre, University of Southampton and University Hospital Southampton NHS Foundation Trust, and NIHR Musculoskeletal Biomedical Research Unit, University of Oxford. The work leading to these results was supported by the European Union's Seventh Framework Programme (FP7/2007-2013), projects EarlyNutrition and ODIN under grant agreements numbers 289346 and 613977.

\section{Compliance with ethical standards}

Conflict of interest None. 
Open Access This article is distributed under the terms of the Creative Commons Attribution 4.0 International License (http:// creativecommons.org/licenses/by/4.0/), which permits unrestricted use, distribution, and reproduction in any medium, provided you give appropriate credit to the original author(s) and the source, provide a link to the Creative Commons license, and indicate if changes were made.

\section{References}

1. US National Library of Medicine National Institutes of Health. Pubmed.gov (2016) https://www.ncbi.nlm.nih.gov/pubmed/? term=vitamin+D. Accessed 20/11/2016

2. Theodoratou E, Tzoulaki I, Zgaga L, Ioannidis JP (2014) Vitamin D and multiple health outcomes: umbrella review of systematic reviews and meta-analyses of observational studies and randomised trials. BMJ 348:g2035. doi:10.1136/bmj.g2035

3. Harvey N, Holroyd C, Ntani G, Javaid M, Cooper P, Moon R et al (2014) Vitamin D supplementation in pregnancy: a systematic review. Health Technol Assess 18(45):1-190. doi:10.3310/hta18450

4. Schottker B, Jorde R, Peasey A, Thorand B, Jansen EH, Groot L et al (2014) Vitamin D and mortality: meta-analysis of individual participant data from a large consortium of cohort studies from Europe and the United States. BMJ 348:g3656. doi:10.1136/bmj. g3656

5. Bilinski K, Boyages S (2013) Evidence of overtesting for vitamin D in Australia: an analysis of 4.5 years of Medicare Benefits Schedule (MBS) data. BMJ Open 3(6):e002955. doi:10.1136/bmjopen-2013002955

6. Kotta S, Gadhvi D, Jakeways N, Saeed M, Sohanpal R, Hull S et al (2015) "Test me and treat me"-attitudes to vitamin D deficiency and supplementation: a qualitative study. BMJ Open 5(7):e007401. doi:10.1136/bmjopen-2014-007401

7. Holick MF, Binkley NC, Bischoff-Ferrari HA, Gordon CM, Hanley DA, Heaney RP et al (2011) Evaluation, treatment, and prevention of vitamin D deficiency: an Endocrine Society clinical practice guideline. J Clin Endocrinol Metab 96(7):1911-1930. doi:10. 1210/jc.2011-0385

8. National Institute for Health and Care Excellence (2014) Vitamin D: increasing supplement use in at-risk groups [PHC56]. https:// www.nice.org.uk/guidance/ph56
9. Ross AC, Taylor CL, Yaktine AL, Del Valle HB (2011) Dietary Reference Intakes for Calcium and Vitamin D

10. Deschasaux M, Souberbielle JC, Partula V, Lecuyer L, Gonzalez R, Srour B et al. (2016) What Do People Know and Believe about Vitamin D? Nutrients. 8(11). doi:10.3390/nu8110718

11. Bonevski B, Bryant J, Lambert S, Brozek I, Rock V (2013) The $\mathrm{ABC}$ of vitamin D: a qualitative study of the knowledge and attitudes regarding vitamin $\mathrm{D}$ deficiency amongst selected population groups. Nutrients 5(3):915-927. doi:10.3390/nu5030915

12. Toher C, Lindsay K, McKenna M, Kilbane M, Curran S, Harrington L et al (2014) Relationship between vitamin D knowledge and 25-hydroxyvitamin D levels amongst pregnant women. J Human Nutr Diet 27(3):261-269. doi:10.1111/jhn.12150

13. Hesse BW, Nelson DE, Kreps GL, Croyle RT, Arora NK, Rimer BK et al (2005) Trust and sources of health information: the impact of the Internet and its implications for health care providers: findings from the first Health Information National Trends Survey. Arch Intern Med 165(22):2618-2624. doi:10.1001/archinte.165.22.2618

14. Ginsberg J, Mohebbi MH, Patel RS, Brammer L, Smolinski MS, Brilliant L (2009) Detecting influenza epidemics using search engine query data. Nature 457(7232):1012-1014. doi:10.1038/ nature 07634

15. Carr LJ, Dunsiger SI (2012) Search query data to monitor interest in behavior change: application for public health. PLoS One 7(10): e48158. doi:10.1371/journal.pone.0048158

16. InternetLiveStats. Google Search Statistics. http://www. internetlivestats.com/google-search-statistics/. Accessed 20/11/ 2016

17. Telfer S, Woodburn J (2015) Let me Google that for you: a time series analysis of seasonality in internet search trends for terms related to foot and ankle pain. J Foot Ankle Res 8:27. doi:10. 1186/s13047-015-0074-9

18. Ingram DG, Plante DT (2013) Seasonal trends in restless legs symptomatology: evidence from Internet search query data. Sleep Med 14(12):1364-1368. doi:10.1016/j.sleep.2013.06.016

19. Jha S, Wang Z, Laucis N, Bhattacharyya T (2015) Trends in media reports, oral bisphosphonate prescriptions, and hip fractures 19962012: an ecological analysis. J Bone Miner Res 30(12):2179-2187. doi:10.1002/jbmr.2565

20. Hong YA, Cho J (2016) Has the digital health divide widened? Trends of health-related internet use among older adults from 2003 to 2011. J Gerontol B Psychol Sci Soc Sci. doi:10.1093/ geronb/gbw100 